\title{
Realization and numerical simulation of a high power and high efficiency Holmium doped fiber laser at $2.1 \mu \mathrm{m}$
}

\author{
Julien Le Gouët ${ }^{1}$, François Gustave ${ }^{1}$, Pierre Bourdon ${ }^{1}$, Thierry Robin ${ }^{2}$, Arnaud Laurent ${ }^{2}$, Benoit Cadier $^{2}$ \\ 1. O.N.E.R.A., Palaiseau, France \\ 2. iXblue, Lannion, France
}

Holmium fiber lasers pumped in-band with Tm fiber lasers appear as interesting solutions when looking for high power in a single spatial mode and high electro-optic efficiency, in a spectral domain that guarantees eye-safe emission and low absorption by the atmosphere. We will present the progress of our work on a high efficiency holmium (Ho) fiber laser, detailing the numeric model, experimental realization and results.

In order to realize a fiber-based laser in the $2 \mu \mathrm{m}$ spectral domain, one general concern is the concentration of hydroxyl groups $\mathrm{OH}^{-}$, which present a high and broad absorption peak close to $2200 \mathrm{~nm}$ [1]. As will be introduced, previous experiments performed on in-band core-pumped holmium-doped single-clad fibers indicated that we could obtain very high purity silica core and clad. We estimated there an $\mathrm{OH}$ concentration at least one order of magnitude lower compared to the fibers used for state of the art Ho-fiber laser demonstrations [2].

Another concern for clad pumping at high power $(>100 \mathrm{~W})$ is the absorption of the $1940 \mathrm{~nm}$ pump radiation by the standard polymer coating used in double-clad fibers. A thin external clad of fluorine doped silica must be added to avoid this pump loss, resulting in a so-called triple-clad fiber $(3 \mathrm{CF})$. We designed and manufactured large mode area (LMA) triple-clad fibers (Ho doped and passive), with core and clad diameters of respectively $20 \mu \mathrm{m}$ and $250 \mu \mathrm{m}$, and corresponding numerical apertures (NA) of 0.08 and 0.22 .

These triple-clad fibers are used in an all-fiber laser cavity, as illustrated on Fig. 1 (left): the Ho-doped active medium is enclosed between a fiber Bragg grating (FBG) inscribed in the core of the passive 3CF, and the low reflectivity interface of a normal angle fiber cleave. The FBG is highly reflective $(\mathrm{R}>98 \%)$ at $2120 \mathrm{~nm}$, with a bandwidth of about $2 \mathrm{~nm}$.
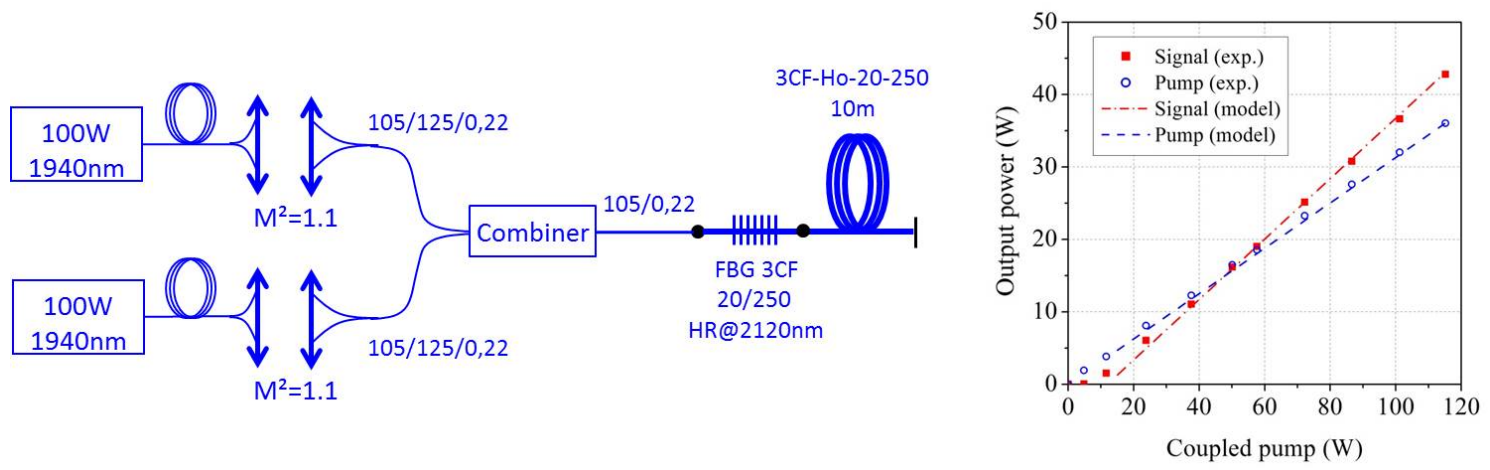

Fig. 1 Left: design of the fiber laser configuration. Right: measurement (dots) and numerical model(lines) for the signal (red) and residual pump (blue) powers at the laser output, as a function of the coupled pump power.

The fiber is pumped by two Tm-fiber lasers, each delivering up to $120 \mathrm{~W}$ at $1940 \mathrm{~nm}$, and combined into a multimode fiber which is spliced to the $3 \mathrm{CF}$ fiber containing the FBG. So far we have run the pump lasers up to $60 \mathrm{~W}$ each, coupling about $110 \mathrm{~W}$ at the cavity input. The resulting efficiency is $40 \%$, with a pump threshold of about $10 \mathrm{~W}$ (Fig. 1, left). The high residual pump power indicates that the optimal fiber length is longer than the $10 \mathrm{~m}$ sample of Ho $3 \mathrm{CF}$ tested at the moment.

Thanks to this first experiment, and thorough spectroscopic studies lead on single clad samples, we could optimize our numerical model for in-band pumped fiber laser (see Fig.1, dotted lines). The short term investigations will consist in using this model to optimize the fiber length and extract the maximum laser efficiency with this fiber. Our work on spectral and time domain analysis will also be presented, as well as the spatial mode characterization. Finally, we will discuss our prospects on a high power fiber amplifier configuration based on this fiber.

[1] O. Humbach, H. Fabian, U. Grzesik, U. Haken, W. Heitmann, "Analysis of OH absorption bands in synthetic silica," J. Non-Cryst. Solids 203, pp. 19-26 (1996).

[2] J. Wang, D. Yeom, N. Simakov, A. Hemming, A. Carter, S. B. Lee, and K. Lee, "Numerical Modeling of in-Band Pumped Ho-Doped Silica Fiber Lasers," Journal of Lightwave Technology 36, pp. 5863-5880 (2018). 